\title{
Too little or too much? Parafoveal preview benefits and parafoveal load costs in dyslexic adults
}

\author{
Susana Silva ${ }^{1,2} \cdot$ Luís Faísca $^{2} \cdot$ Susana Araújo $^{2}$. \\ Luis Casaca $^{2} \cdot$ Loide Carvalho $^{2}$. \\ Karl Magnus Petersson ${ }^{2,3,4}$ - Alexandra Reis ${ }^{2}$
}

Received: 1 May 2015 /Accepted: 12 August 2015 /Published online: 23 September 2015

(C) The International Dyslexia Association 2015

\begin{abstract}
Two different forms of parafoveal dysfunction have been hypothesized as core deficits of dyslexic individuals: reduced parafoveal preview benefits ("too little parafovea") and increased costs of parafoveal load ("too much parafovea"). We tested both hypotheses in a single eye-tracking experiment using a modified serial rapid automatized naming (RAN) task. Comparisons between dyslexic and non-dyslexic adults showed reduced parafoveal preview benefits in dyslexics, without increased costs of parafoveal load. Reduced parafoveal preview benefits were observed in a naming task, but not in a silent letter-finding task, indicating that
\end{abstract}

Susana Silva

zanasilva@gmail.com

Luís Faísca

lfaisca@ualg.pt

Susana Araújo

smaraujo@ualg.pt

Luis Casaca

luismiguelcasaca@gmail.com

Loide Carvalho

loidemcarvalho@gmail.com

Karl Magnus Petersson

kmpetersson@ualg.pt

Alexandra Reis

aireis@ualg.pt

1 Center for Psychology of the University of Porto (CPUP), Faculdade de Psicologia e Ciências da Educação da Universidade do Porto, Rua Alfredo Allen, 4200-135 Porto, Portugal

2 Cognitive Neuroscience Research Group, Department of Psychology and Educational Sciences and Centre for Biomedical Research (CBMR), University of Algarve, 8005-139 Faro, Portugal

3 Max Planck Institute for Psycholinguistics, Nijmegen, The Netherlands

4 Donders Institute for Brain, Cognition and Behaviour, Radboud University Nijmegen, Nijmegen, The Netherlands 
the parafoveal dysfunction may be consequent to the overload with extracting phonological information from orthographic input. Our results suggest that dyslexics' parafoveal dysfunction is not based on strict visuo-attentional factors, but nevertheless they stress the importance of extra-phonological processing. Furthermore, evidence of reduced parafoveal preview benefits in dyslexia may help understand why serial RAN is an important reading predictor in adulthood.

Keywords Dyslexia $\cdot$ Eye-tracking $\cdot$ Parafovea $\cdot$ Reading predictors $\cdot$ Serial RAN

\section{Introduction}

Parafoveal vision captures the visual field surrounding the fovea ( $1^{\circ}$ around the fixation point) up to $5^{\circ}$. In reading, the foveal and the parafoveal text are both part of the perceptual span. While fixating word $\mathrm{N}$, skilled readers are able to preprocess the upcoming word $(\mathrm{N}+1)$. Preprocessing word $\mathrm{N}+1$ may shorten processing times when the word is later fixated, and thus contribute to optimize the reading process. This phenomenon is known as the occurrence of parafoveal preview benefits for word N+1 (Schotter, Angele, \& Rayner, 2012). Parafoveal preview is an opportunity to readers, but it is also an additional stimulus they have to deal with. Therefore, a different perspective on parafoveal vision is that word $\mathrm{N}+1$ is a parafoveal load that taxes the processing of word N (e.g., Moll \& Jones, 2013). In this view, parafoveal load costs impinging upon word $\mathrm{N}$ may be considered.

The idea of a parafoveal dysfunction in dyslexic individuals is a long-standing one (e.g., Bouma \& Legein, 1977), but it is still unclear if it relates to atypical preview benefits or to atypical load costs. The two hypotheses have been put forward to account for findings that dyslexic participants perform more poorly in serial rapid automatized naming tasks (RAN, Denckla \& Rudel, 1976) than in single-item naming tasks (discrete RAN), contrasting with the improved performance of non-dyslexics in serial RAN (Jones, Branigan, \& Kelly, 2009; Zoccolotti et al., 2013). Zoccolotti and colleagues (2013) suggested that dyslexic participants might have ignored the parafoveal input in serial RAN, thus putting forward the hypothesis of reduced parafoveal preview benefits ("too little parafovea," in our own phrasing). In contrast, Jones and collaborators (2009) claimed that dyslexic participants might have been confused by the presence of parafoveal input in serial RAN, suggesting that dyslexic individuals suffer increased parafoveal load costs compared to healthy ones ("too much parafovea"). These two hypotheses draw upon previous literature findings, and their relevance goes beyond contributing to explain why individuals with dyslexia are impaired in serial RAN tasks. As we are about to show, comparing the hypotheses of reduced parafoveal preview benefits and increased parafoveal load costs may also shed light on the weight of visuo-attentional vs. phonological factors in dyslexia (e.g., Ziegler, Pech-Georgel, Dufau, \& Grainger, 2010), specifically on how the parafoveal dysfunction stems from each of these two kinds of deficits.

The first hypothesis, stating that dyslexic individuals have reduced parafoveal preview benefits ("too little parafovea"), has been supported by a few studies (Rayner, Murphy, Henderson, \& Pollatsek, 1989; Yan, Pan, Laubrock, Kliegl, \& Shu, 2013) using gazecontingent paradigms (McConkie \& Rayner, 1975) that manipulate the amount of parafoveal information made available at a given point. The hypothesis has been articulated as a problem of overload with the currently fixated, foveal item (item N). 
Specifically, individuals with dyslexia may be overloaded while extracting phonological information from the fixated orthographic input, and they may consequently lack resources to preprocess the parafoveal item (Jones, Kelly, \& Corley, 2007; Yan et al., 2013; Zoccolotti et al., 2013). The idea of overload with the foveal item is consistent with evidence that text difficulty reduces the perceptual span (Henderson \& Ferreira, 1990), as well as with evidence of lower parafoveal preview benefits in less skilled readers (Chace, Rayner, \& Well, 2005; Sperlich, Schad, \& Laubrock, 2015). All these findings fit into the EZ-reader model of eye movement control in reading (Pollatsek, Reichle, \& Rayner, 2006; Reichle, Rayner, \& Pollatsek, 2003), which predicts that the time left for parafoveal preview decreases as the processing of the currently fixated word takes longer. The idea of overload with the phonology of the foveal item frames the parafoveal dysfunction as a consequence of the reading problem and it partly acknowledges the weight of phonological factors in dyslexia, but it is not the only approach possible. An alternative way of articulating the hypothesis of reduced parafoveal preview benefits would be admitting that the lack of preview reflects a primary visuo-attentional deficit, with no need to consider phonological deficits. Although this way of articulating the "too little parafovea" hypothesis is not explicit in the literature, it would be consistent with evidence of visuo-attentional impairments in dyslexia (Bellocchi, Muneaux, BastienToniazzo, \& Ducrot, 2013; Lewandowska, Milner, Ganc, Włodarczyk, \& Skarżyński, 2014), particularly with evidence of a reduced visual attention span (Bosse, Tainturier, \& Valdois, 2007; Germano, Reilhac, Capellini, \& Valdois, 2014; Hawelka \& Wimmer, 2005; Valdois, Bosse, \& Tainturier, 2004).

The second hypothesis ("too much parafovea"), stating that parafoveal input is a load with increased costs to dyslexics (Jones et al., 2009) has been linked to crowding phenomena. Crowding (Bouma, 1973) occurs when the processing of the currently fixated item is impaired by adjacent (flanking) items. Crowding may be observed in healthy individuals, but increased crowding effects have been found in dyslexic ones, both when the clustered items lie at the fovea (Bouma \& Legein, 1977; Callens, Whitney, Tops, \& Brysbaert, 2013; Martelli, Di Filippo, Spinelli, \& Zoccolotti, 2009; Moores, Cassim, \& Talcott, 2011; Spinelli, De Luca, Judica, \& Zoccolotti, 2002) and at the parafovea (Bouma \& Legein, 1977; Martelli et al., 2009; Pernet, Valdois, Celsis, \& Démonet, 2006). In line with this, it has been shown that an increased inter-letter spacing improves reading performance in dyslexics more than it does in non-dyslexics (Perea, Panadero, Moret-Tatay, \& Gómez, 2012; Spinelli et al., 2002; Zorzi et al., 2012). Critical to the argument of a costly parafoveal load (Jones et al., 2009), the crowding span of dyslexics seems to be larger than that of non-dyslexics, such that the processing of a flanked foveal item by dyslexics is hampered even when the flanking item lies at parafoveal distance, that is, when the two items are separated by more than $1^{\circ}$ (Jones, Obregón, Louise Kelly, \& Branigan, 2008; Moll \& Jones, 2013; Pernet et al., 2006). Although crowding effects pertain to the visuo-attentional domain (Bellocchi et al., 2013), it has been suggested that the presence of flanking items is not critical to visual processing per se, but rather to the extraction of phonological information (Hawelka \& Wimmer, 2008; Jones et al., 2008). Therefore, two different ways of articulating the hypothesis of increased parafoveal load costs also seem to exist: one involving phonological processing deficits (crowding affects the extraction of phonological information) and the other not (crowding as purely visuo-attentional).

To our knowledge, the hypothesis of reduced parafoveal preview benefits ("too little parafovea") has not yet been tested against that of increased parafoveal load costs ("too much 
parafovea"). One of the reasons for this may be that parafoveal preview benefits and parafoveal load costs are not easily disentangled. Manipulating the amount of available parafoveal information usually leads to variations in both preview and load: a previewable parafoveal item is also a parafoveal load, and vice-versa. This is valid for gaze-contingent paradigms, which have provided evidence of reduced parafoveal preview benefits (Rayner et al., 1989; Yan et al., 2013). This is also valid for paradigms such as that implemented by Moll and Jones (2013), who compared two types of RAN matrices, one with foveally spaced items and another with parafoveally spaced ones. The authors found that, unlike control participants, dyslexics did not improve performance in matrices with parafoveally spaced items and concluded that dyslexics had an atypically large (parafoveal) crowding span. Nevertheless, an explanation based on preview benefits cannot be ruled out: dyslexics may not have benefited from the parafoveal preview of the adjacent item as it became available (parafoveally spaced condition), while control participants may have benefited, thus improving their performance. Therefore, the goal of the present study was to test the hypothesis of reduced parafoveal preview benefits against that of increased parafoveal load costs in a single experiment so as to further understand dyslexics' parafoveal dysfunction, if there is one. To that purpose, we carried out an eye-tracking experiment where a group of adults with dyslexia was compared to a group of non-dyslexics while naming multiple items of a modified serial RAN task. In this naming task, we manipulated the parafoveal load and the parafoveal preview potential of each item in a $2 \times 2$ design that allowed us to obtain independent measures of the parafoveal load cost (increased processing time for the fixated item $\mathrm{N}$, when followed by item $\mathrm{N}+1$ in the parafovea) and the parafoveal preview benefit (decreased processing time for item $\mathrm{N}$ when it stands on the right parafovea of item N-1). At this level, we wanted to determine in which of the two parameters dyslexic participants differ from non-dyslexic ones. Since parafoveal preview skills develop throughout childhood (Sperlich et al., 2015), reduced parafoveal preview benefits are more likely to be detected in adults. For this reason, we compared adults with dyslexia with healthy ones.

It was also our goal to determine whether or not phonology is involved in each of the two hypothetical parafoveal dysfunctions, since, as we saw, each may be articulated either at a purely visuo-attentional level or at a phonology-dependent one. To that purpose, we created a second eye-tracking task, similar to the previous one but where naming was suppressed and replaced by a silent letter-finding instruction, such that participants were freed from the extraction of phonological information. If there is a reduced parafoveal preview benefit and if it is more prominent in the naming task than in the letter-finding task, then the reduced benefit may be a consequence of the overload caused by extracting phonological information. Conversely, if there are no cross-task differences, then the reduced benefit may be a visuoattentional cause of the reading difficulties. If dyslexics show an increased load cost and it is more prominent in the naming task, this means that the parafoveal input leads to crowding only when there is extraction of phonological information. Otherwise, there will be support for strictly visuo-attentional parafoveal crowding. Our silent letter-finding task may be viewed as a visual search task. Visual search skills have been probed in dyslexic and non-dyslexic participants with mixed findings. While Wimmer, Mayringer, and Landerl (1998) found similar performance levels in the two groups, the performance of dyslexic participants was impaired in the study of Di Filippo et al. (2006). Moreover, visual search performance was found to be a weaker predictor of reading performance than rapid automatized naming (Di Filippo et al., 2005, 2006; Georgiou, Parrila, Cui, Papadopoulos, 2013). In this sense, we may expect smaller cross-group differences in the silent letter-finding task than in naming at the 
level of general performance, but that does not necessarily mean that the parafoveal preview benefits or the parafoveal load costs of dyslexics vs. non-dyslexics are equivalent in the silent letter-finding task.

\section{Materials and methods}

\section{Participants}

Seventeen dyslexic adults, and 17 normal-readers took part in the experiment, after being profiled for reading and reading-related skills (Table 1). All dyslexic participants were native Portuguese speakers and they had a history of reading disorder expressed by scores below the cut-off point (35) on a self-report measure of reading history (QHL; Castro \& Alves, 2005), a formal diagnosis of reading and spelling problems as a child, non-verbal intelligence measured by WAIS III (Wechsler 1997, Portuguese norms by Barreto, Moreira, \& Ferreira, 2008) within normal-range, and test results (see below, reading and reading-related measures) indicative of adult reading problems (1.5 SD below the mean). None had neurological or oral language problems. Dyslexic participants were matched for sex, age, and schooling with 17 nondyslexic control participants. The latter were native Portuguese speakers without any history, formal diagnosis, or test results indicating reading disorders, with no neurological or oral language problems, and with normal-range non-verbal intelligence. The two groups differed

Table 1 Profiles of dyslexic vs. non-dyslexic participants

\begin{tabular}{|c|c|c|c|c|}
\hline & $\begin{array}{l}\text { Dyslexics } \\
n=17 \\
F=10 ; M=7\end{array}$ & $\begin{array}{l}\text { Non-dyslexics } \\
n=17 \\
F=10 ; M=7\end{array}$ & $p$ & Cohen's $d$ \\
\hline Age & $26.35 \pm 8.41$ & $26.12 \pm 6.92$ & Ns & 0.03 \\
\hline Years of schooling & $14.65 \pm 2.40$ & $15.18 \pm 2.24$ & Ns & -0.23 \\
\hline QHL (max. 100) & $65.84 \pm 10.89$ & $31.53 \pm 9.52$ & .000 & 1.70 \\
\hline \multicolumn{5}{|l|}{ Cognitive measures } \\
\hline Non-verbal IQ WAIS III & $107.35 \pm 12.81$ & $114.41 \pm 9.47$ & Ns & -0.59 \\
\hline \multicolumn{5}{|l|}{ Reading measures } \\
\hline Words-accuracy (\%) & $97.41 \pm 2.88$ & $99.55 \pm 0.63$ & .008 & -0.92 \\
\hline Words-time (w/s) & $1.33 \pm 0.22$ & $1.93 \pm 0.30$ & .000 & -1.51 \\
\hline Pseudowords-accuracy (\%) & $95.29 \pm 7.42$ & $98.66 \pm 2.14$ & Ns & -0.60 \\
\hline Pseudowords-time (w/s) & $0.90 \pm 0.21$ & $1.52 \pm 0.25$ & .000 & -1.61 \\
\hline Reading comprehension (TIL, max. 36) & $10.35 \pm 1.94$ & $16.82 \pm 3.97$ & .000 & -1.44 \\
\hline \multicolumn{5}{|l|}{ Reading-related measures } \\
\hline Phoneme deletion (max. 18) & $13.53 \pm 3.28$ & $17.59 \pm 0.71$ & .000 & -1.30 \\
\hline Spoonerism (max. 38) & $22.88 \pm 12.42$ & $35.06 \pm 5.45$ & .001 & -1.07 \\
\hline RAN letters (items/s) & $2.03 \pm 0.44$ & $2.87 \pm 0.43$ & .000 & -1.39 \\
\hline RAN digits (items/s) & $2.20 \pm 0.45$ & $3.08 \pm 0.57$ & .000 & -1.30 \\
\hline Digit span WAIS III (SS $\left.{ }^{\mathrm{a}}\right)$ & $8.65 \pm 3.28$ & $11.71 \pm 2.97$ & .008 & -0.89 \\
\hline Vocabulary WAIS III (SS) & $10.41 \pm 2.62$ & $12.11 \pm 2.42$ & Ns & -0.64 \\
\hline
\end{tabular}

${ }^{\text {a }}$ Standard scores 
significantly in all reading and reading-related measures, except for pseudoword reading accuracy and vocabulary (Table 1), where differences were marginal.

\section{Stimuli}

\section{Reading and reading-related measures}

Tests of reading skills included an adaptation of 3DM (Blomert \& Vaessen, 2009) to the Portuguese population (Reis et al., 2014), measuring the accuracy and speed of word and pseudoword reading, and a test of reading comprehension (1-min TIL; Fernandes, Araújo, Sucena, Reis, \& Castro, 2014; Sucena \& Castro, 2010). In the 3DM test, participants are presented with 75 low-frequency words, 75 high-frequency words, and 75 pseudowords for a fixed time interval and their task is to name as many words or pseudowords as possible. In the TIL test, participants are required to read silently an incomplete sentence and to choose, among four options, the word that best fits into the context. Measures of reading-related skills were obtained from two phonological awareness tasks (phoneme deletion and spoonerism), from traditional serial RAN tasks $(5 \times 10$ items, letter version and digit version $)$ and from the digit span and vocabulary tests of WAIS III.

\section{Modified RAN task}

The stimulus set was composed of 28 RAN matrices containing letters (a o s p d). All letters occurred approximately with the same frequency and they were pseudorandomly ordered such that there were no immediate repetitions. The spatial arrangement of the traditional $5 \times 10$-item matrix (Denckla \& Rudel, 1976) was modified, such that letter items were irregularly spaced within each row (Fig. 1). Half the letter items stood in the right parafoveal span of the preceding item (inter-letter space of $2.86^{\circ}$ ) and thus they could be parafoveally previewed (P). The other half stood beyond the parafoveal span of N-1 (inter-letter space of $8.57^{\circ}$, non-previewed, $\mathrm{nP}$ ). Half the items were followed by parafoveal inputs at the right (had parafoveal load, L) and the other half were not (no parafoveal load, $\mathrm{nL}$ ). There were, thus, four classes of items (PL, PnL, $\mathrm{nPL}, \mathrm{nPnL}$ ) with 98 letters each, and this distribution replicated for each letter type (a o s p d),

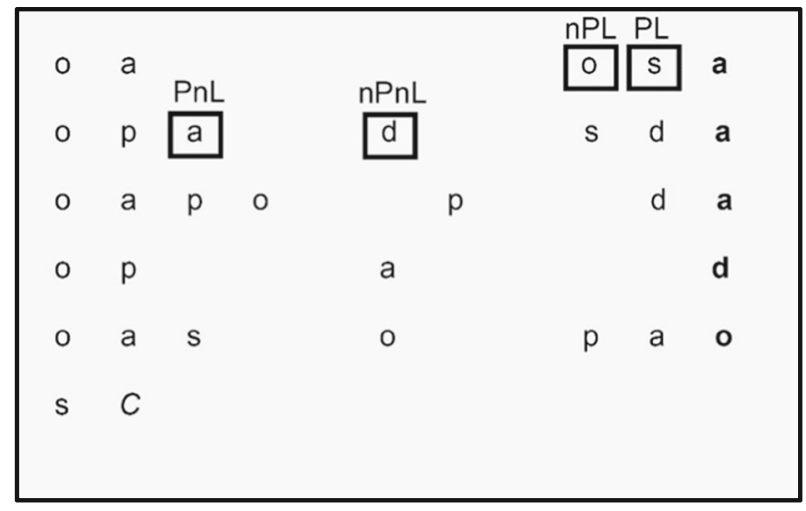

Fig. 1 Example of a modified RAN matrix, with row types 1, 2, 3, 5, 2 (see text for details). Examples of each item class are highlighted ( $n P L$ non-previewed item with load, $P L$ previewed item with load, $P n L$ previewed item with no load, $n P n L$ non-previewed item with no load) 
corresponding to a total of 392 items. We created six row types, each containing a particular combination of items from different classes bounded by filler (F) items on the left and on the right (type 1: F-F-nPL-PL-F, type 2: F-F-PnL-nPnL-nPL-PL-F, type 3: F-F-PL-PnL-nPnL-nPLF, type 4: F-F-PnL-nPnL-F, type 5: F-F-PnL-F, type 6: F-F-nPnL-F). The last row in each matrix was always composed of two filler items (F-F), with the second one italicized in order to remind participants to move on to the next matrix. Each matrix contained a different combination of row types, such that the geometry of the matrix was non-predictable.

\section{Procedure}

Participants performed a letter-naming task (task 1) and a letter-finding task (task 2) on the same stimulus set, while their eye movements were recorded. In task 1, participants named the letters in rows as fast as possible. In task 2 , they were requested to look silently at the items and say yes or no when reaching the end of each row, depending on whether they saw or not the sequence "s-p" in that row. The order of the tasks was counterbalanced, and both included a practice session in the beginning. The eye movements were monocularly recorded with the SMI Hi-speed 1250-Hz system (http://www.smivision.com/). Participants sat $80 \mathrm{~cm}$ away from the stimulus monitor and placed their head on a chin rest. Before each task, participants were given a series of practice trials. A 5-point calibration procedure followed. Tracking errors larger than $0.5^{\circ}$ were rejected and followed by new calibration procedures. In both tasks, participants were asked to stand still with their right hand over the computer keyboard and to press the enter key when reaching the last (italicized) letter of the screen. Vocal responses were continuously recorded with a webcam connected to the system.

At the end of the session, participants performed a control letter-finding task with no eye movements recorded (task 3 ). The task was designed to rule out any differences in letterfinding (sequencing) abilities that might account for differences in task 2 . In this control letterfinding task, participants saw 30 sequences with letters presented one at a time (5-12 letters per sequence) and with inter-stimulus intervals of $330 \mathrm{~ms}$. Similar to task 2, they were requested to detect whether a two-letter sequence was present. They did it by pressing either a yes or a no button at the end of each sequence, paralleling the procedure in task 2 (respond when reaching the end of the row).

\section{Preprocessing of eye-tracking data}

Items with incorrect responses and matrices with artifacts (blinks and signal losses) exceeding $30 \%$ of trial time were removed from the analysis. On average, $4.5 \%$ of the items were excluded in task 1 and $2.2 \%$ in task 2 . Events were extracted based on a high-speed algorithm for saccade detection. A threshold of $30^{\circ}$ peak velocity and a minimum duration of $22 \mathrm{~ms}$ defined a saccade. Fixations shorter than $50 \mathrm{~ms}$ were rejected. Rectangular areas were placed around each letter item and labeled according to the condition (F, PL, PnL, nPL, nPnL), defining the areas of interest (AOIs) on which eye-tracking parameters were computed.

\section{Statistical analysis}

Average fixation times per letter were obtained for all items (global fixation times) and for each item class separately (PL, PnL, nPL, nPnL) in both tasks. First, the correlation between global fixation times in the experimental (modified) RAN task and the behavioral performance in the 
traditional RAN used for profiling was computed, so as to grant that our modified version kept the essential mechanisms of serial RAN. The hypotheses of reduced parafoveal preview benefit vs. increased parafoveal load cost were then tested for each task separately (task 1naming, task 2-letter finding). Hypotheses were tested in two different ways. First, we ran mixed ANOVAs with parafoveal preview potential (PREVIEW, 2 levels, P vs. nP) and parafoveal load (LOAD, 2 levels, L vs. $\mathrm{nL}$ ) as within-subjects factors and with GROUP as between-subjects factor. Additionally, we compared the parafoveal preview benefit and the parafoveal load cost of dyslexic vs. control participants. The parafoveal preview benefit was computed with the formula (non-previewed - previewed)/previewed, indicating the proportion of the fixation time for previewed items $(\mathrm{PL}+\mathrm{PnL})$ that was additionally required when looking at items with no preview (nPL $+\mathrm{nPnL})$. Similarly, the parafoveal load cost was computed as the proportion of the fixation time for non-loaded items $(\mathrm{PnL}+\mathrm{nPnL})$ that was additionally required when there was a parafoveal load $(\mathrm{PL}+\mathrm{nPL})$. The formula was (loaded-non-loaded)/non-loaded. The presence of parafoveal preview benefits and parafoveal load costs was determined by one-sample $t$ tests against zero values, and differences between groups were analyzed with independent-samples $t$ tests. The two hypothesis testing approaches tap essentially into the same question, but the $2 \times 2$ factorial approach emphasizes the direct contrast between preview and load effects, while the approach based on parafoveal preview benefit and parafoveal load cost uses relative measures (proportions) that emphasize the notions of benefit and cost. The parafoveal preview benefit and the parafoveal load cost of all participants were correlated with reading measures. Participants were compared for accuracy and reaction time in task 3 , by means of independent-samples $t$ tests. Unless otherwise specified, a significance level of .05 was adopted.

\section{Results}

\section{Letter-finding control task (task 3)}

Accuracy was near $100 \%$ in both groups (max. 30, $M \pm \mathrm{SD}$ : dyslexics $=29.7 \pm 0.77$, nondyslexics $=30 \pm 0.46)$, no significant differences being observed $(p>.21, d=-0.51)$. Reaction times did not differ across groups either (dyslexics $=589 \mathrm{~ms} \pm 98$, non-dyslexics $=606 \mathrm{~ms} \pm 107$, $p>$.66). The fact that the two groups were similar in their ability to detect a sequence of two letters when these are presented in isolation helps ruling out the possibility that any group differences in task 2 are due to letter sequencing problems in dyslexics, instead of being due to the parafoveal processes we were interested in. However, given the ceiling effects for accuracy, we decided to be cautious and use it merely as a gross indicator of sequencing abilities in case it is necessary, that is, if the two groups differ in task 2.

\section{Correlation between traditional RAN and modified RAN}

Performance in the traditional letter RAN task (mean + SD: dyslexics $=2.87 \pm 0.43$, non-dyslexics $=2.03 \pm 0.44$ ) correlated with the average fixation time per letter in task 1 (dyslexics= $242 \pm 78$, non-dyslexics $=187 \pm 37, r=-.535, p=.001)$ and, less strongly, in task 2 (dyslexics $=$ $161 \pm 56$, non-dyslexics $=151 \pm 39, r=-.346, p=.045$ ). The basic mechanisms of the traditional serial RAN task seem thus to be preserved in our modified version. Not surprisingly, the absence of naming requirements in task 2 weakened the association. 


\section{Effects of parafoveal preview potential and parafoveal load cost on fixation time}

In task 1 (naming, see Fig. 2), dyslexics had longer fixation times than non-dyslexics $(F(1,32)=$ 16.4, $\left.p<.001, \eta_{p}^{2}=.339\right)$. The interaction between GROUP and PREVIEW was marginally significant $\left(F(1,32)=3.2, p=.084, \eta_{p}^{2}=.090\right)$, indicating a significant effect $(\mathrm{nP}>\mathrm{P})$ in nondyslexics $\left(F(1,16)=15.3, p=.001, \eta_{p}^{2}=.489\right)$, but not in dyslexics $(p>.14)$. There was a main effect of LOAD (L>nL: $\left.F(1,32)=13.6, p=.001, \eta_{p}^{2}=.298\right)$, and the interaction between PREVIEW and LOAD was non-significant $(p>.79)$. Neither LOAD nor PREVIEW $\times$ LOAD interacted further with GROUP $(p>.26)$.

In task 2 (letter finding), the fixation times of the two groups did not differ $(p>.28)$. There were no interactions of within-subjects factors with GROUP $(p>.57)$. A significant PREVIEW $\times$ LOAD interaction $\left(F(1,32)=67.6, p<.001, \eta_{p}^{2}=.619\right.$, Fig. 2) indicated that, for both groups, fixation times on loaded $(\mathrm{L})$ items increased when preview was possible $(\mathrm{P})$, while fixation times on non-loaded items $(\mathrm{nL})$ decreased with preview. Thus, only non-loaded items elicited a preview benefit-like pattern.

\section{Parafoveal preview benefit and parafoveal load cost}

In the naming task, the parafoveal preview benefit (Fig. 3) of non-dyslexics was significant $(t(16)=5.28, p<.001)$ and that of dyslexics was marginal $(t(16)=1.77, p=.096)$. In the letter-finding task, no group showed a significant parafoveal preview benefit (nondyslexics: $p>.73$, dyslexics: $p>.98)$. Non-dyslexics showed a larger parafoveal preview benefit than dyslexics in the naming task $(t(32)=-2.97, p=.006, d=-0.92)$, but not in the letter-finding task $(p>.79, d=0.09)$. For the whole sample, the parafoveal preview benefit in the naming task correlated significantly with word reading speed $(r=.379, p=.027)$ and word reading accuracy $(r=.377, p=.028)$. The correlation with pseudoword reading speed was marginal $(r=.311, p=.073)$.


DYSLEXIC NON-DYSLEXIC

TASK 2 (LETTER FINDING)

Fig. 2 Average fixation time across levels of parafoveal preview potential ( $P$ previewed, $n P$ non-previewed) and parafoveal load ( $L$ loaded, $n L$ non-loaded). Error bars represent the standard error of the mean 


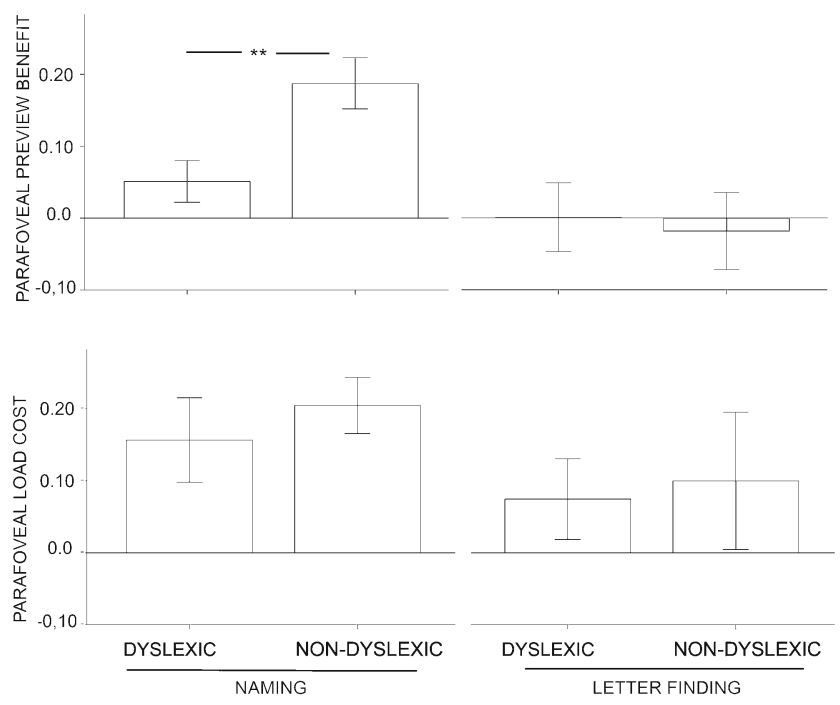

Fig. 3 Parafoveal preview benefit (additional time required to process non-previewed items, expressed as a proportion of the fixation time used for previewed items) and parafoveal load costs (additional time required to process loaded items, expressed as a proportion of the fixation time used for non-loaded items) across groups and tasks. Error bars represent the standard error of the mean

The parafoveal load cost (Fig. 3) was significant for both groups in the naming task (nondyslexics: $t(16)=5.28, p<.001$; dyslexics: $t(16)=2.67, p=.017)$ and non-significant in the letter-finding task $(p>.20)$. There were no cross-group differences, either in the naming task $(p>.50, d=-0.23)$ or in the letter-finding task $(p>.82, d=-0.08)$. There were no significant correlations between parafoveal load cost and reading speed or reading accuracy measures.

\section{Discussion}

In this study, we aimed to test for two hypothesized forms of parafoveal dysfunction in adults with dyslexia: reduced parafoveal preview benefits and increased costs of parafoveal load. Comparisons between dyslexic and non-dyslexic participants only yielded evidence of reduced parafoveal preview benefits. In addition, parafoveal preview benefits correlated moderately with word reading fluency and word decoding in the whole sample, thus strengthening the relation between the parafoveal dysfunction and reading performance.

The absence of group differences in parafoveal load costs suggests that previous findings of a parafoveal crowding span in dyslexic participants but not in healthy ones (Jones et al., 2008; Moll \& Jones, 2013; Pernet et al., 2006) may have resulted from a confound between load and preview. In these studies, the parafoveal input may not have worked as a source of interference (load) on the foveal item, as it was intended, but rather as an underexplored resource (nonpreviewed item) that rendered the performance of dyslexic participants less efficient compared to non-dyslexic controls.

Reduced parafoveal preview benefits for dyslexics showed up in the naming task, but not in the silent letter-finding task. Considering the framework we adopted, which included two different ways of articulating the reduced parafoveal preview hypothesis (visuo-attentional vs. 
phonology-dependent), our findings are in agreement with the phonology-dependent hypothesis, stating that dyslexics do not allocate attentional resources to parafoveal input because they are overloaded with extracting phonological information from orthographic input. Although this way of articulating the reduced parafoveal preview hypothesis acknowledges the weight of phonological factors, in our view such an approach should not be considered a strictly phonological one. The extraction of phonological information from orthographic input corresponds to a component process that the multicomponential view of serial RAN (Norton \& Wolf, 2012) has proposed to be non-strictly phonological (Wolf \& Bowers, 1999). Extracting phonology from orthographic input refers to a process wherein two levels of information are matched, phonology being one of these levels. Problems may be located, for instance, in the matching process itself. Concerning the alternative hypothesis, that reduced parafoveal preview benefits were due to visuo-attentional deficits impeding the access to parafoveal input, our findings did not support it because there were no differences in visual search. However, they were not able to rule it out either because visual search did not elicit significant parafoveal preview benefits in any group. The fact that non-dyslexics did not benefit from parafoveal preview in visual search suggests that parafoveal preview was not a task-relevant mechanism, and the idea of functional (preserved) preview mechanisms in dyslexics is not properly supported when such mechanisms do not seem to be operating in healthy participants. A challenge to future studies may be, thus, devising a phonology-free, RAN-equivalent task in which non-dyslexic participants show significant parafoveal preview benefits, indicating that these benefits are task-relevant and providing proper grounds to determine the role of phonology in generating a parafoveal dysfunction.

Similar cross-group parafoveal load costs in naming as well as in visual search suggest that dyslexic participants do not show exacerbated parafoveal crowding (visuo-attentional) effects, whether or not phonology is involved. The fact that dyslexics' parafoveal dysfunction does not stem from crowding-based visuo-attentional factors does not imply, however, that there are no exacerbated crowding effects in dyslexics (e.g., Moores et al., 2011). In our study, we only excluded the hypothesis of an abnormal (parafoveal) crowding span, according to which a parafoveal item (the parafoveal load) hampers the processing of a foveal one. Crowding effects related to more closely spaced items (item clusters within the fovea or within the parafovea) have not been tested here, since these fall outside of our object of interest, which is the parafoveal load.

Concerning parafoveal load costs, there are two topics deserving further mention. First, despite the absence of group differences in parafoveal load costs, these costs were nonsignificant for all participants when they were freed from extracting phonological information in the letter-finding task (task 2). This may indicate that a parafoveal load is costly only when phonological processes co-occur, as previously suggested (Hawelka \& Wimmer, 2008), and this mechanism may be shared between dyslexics and non-dyslexics. Second, even though dyslexics did not show increased load costs compared to controls, they showed significant load costs in the naming task, just as controls did. Apparently, this is at odds with the idea that parafoveal input is ignored, but one possibility is that dyslexic participants ignore the parafoveal input as a preprocessing target (leading to the reduced preview benefits), but not as a visual stimulus causing interference (crowding). The idea of crowding by a parafoveal item does not necessarily imply an attempt to preprocess that item. Thus, dyslexic participants may have suffered the same amount of crowding as controls, while doing less preprocessing.

Our main findings were that dyslexic adults have reduced parafoveal preview benefits but no increased parafoveal load costs. This pattern was apparent in the context of a modified 
letter-RAN task, in which dyslexic participants were impaired (see the effect of group on fixation times for all letters). Different levels of impairment in serial RAN compared to discrete naming have been observed for different stimulus types (Zoccolotti et al., 2013), and an important question concerns the extent to which our findings would replicate when using stimuli other than single letters. On the one hand, the idea that reduced parafoveal preview benefits may be a consequence of overload with phonology extraction, suggests that the preview benefits in dyslexics may be even more reduced for words, since these require more complex phonological processing. On the other hand, it is possible that increased parafoveal load costs emerge in dyslexic participants when they are confronted with words. The closely spaced letters of words potentiate short-span crowding (inter-letter crowding), to which dyslexic readers seem to be sensitive (e.g., Spinelli et al., 2002), and this might stimulate the parafoveal crowding potential of RAN tasks (inter-word crowding, in this case). Another possibility is that increased parafoveal load costs emerge for words because the simultaneously presented letters in words challenge the visual attention span of dyslexic participants (e.g., Lassus-Sangosse, N'guyen-Morel, \& Valdois, 2008). These hypotheses should be tested in future studies.

Evidence of reduced parafoveal preview benefits in individuals with dyslexia may contribute to explain why they are impaired in serial RAN tasks. Since we did not test for other sources of impairment in serial RAN, we cannot estimate how much this type of parafoveal dysfunction explains the performance disadvantages of dyslexic participants in these tasks. Evidence of reduced parafoveal preview benefits in adults with dyslexia may also be relevant to answer a slightly different question, namely why serial RAN predicts reading ability in adults better than it does in children (Nergård-Nilssen \& Hulme, 2014). Since parafoveal preview benefits are acquired only after some years of reading experience (Sperlich et al., 2015), it is possible that the parafoveal preview benefits of typically developing children are not strong enough to highlight disadvantages in dyslexic children, at least in a way comparable to adults. Future investigations on the parafoveal preview benefits of children and adults with and without dyslexia may shed light on this.

Within the investigation of eye movements in dyslexia, the idea that eye movements reflect atypical cognitive processing (e.g., De Luca, Di Pace, Judica, Spinelli, \& Zoccolotti, 1999; Yan et al., 2013) has been replacing an early hypothesis of a purely oculomotor deficit, such as one related to muscular control in saccade execution and leading to atypical landing positions (Pavlidis, 1981). We did not test for the oculomotor control abilities of our dyslexic participants, but we found evidence of a dysfunction that is not easily explained by saccade execution deficits. On the contrary, reduced parafoveal preview benefits likely explain problems in saccade execution: the inability to collect accurate information on the spatial characteristics of the parafoveal item may impede the efficient planning of a saccade and, thus, its optimal execution shown by typical landing positions.

A frequent methodological concern in dyslexia research relates to the use of chronological age controls, an option we did in the present study. Unlike studies using reading level controls, studies with chronological age controls are likely to miss the effects of reading experience, such that a deficit that is interpreted as a cause of the reading problem is simply a consequence of the smaller reading experience of dyslexic participants. Considering our findings, this specific drawback does not seem to be critical, since they support a view according to which the parafoveal dysfunction of dyslexics is already a consequence of the reading problem (lack of preview by overload with the foveal item), and not a cause. Nevertheless, the possibility that the lack of preview does not stem from an overload with the foveal item, but simply from the 
lack of reading experience, should not be ruled out. Future studies might, thus, compare dyslexic participants with reading-level controls. In any case, the idea that reduced parafoveal preview benefits signal dyslexia and parafoveal load costs do not, remains valid.

\section{Conclusion}

Our study was novel in disentangling the effects of parafoveal preview potential from those of parafoveal load. With this approach, we contributed to specify the parafoveal dysfunction of adults with dyslexia. Our findings strengthen the idea that a reduced parafoveal preview benefit is a core deficit in dyslexia that is consequent to reading problems, and they rule out the possibility that dyslexics' parafoveal dysfunction reflects a crowding-based visuo-attentional deficit.

Acknowledgments This work was supported by Fundação para a Ciência e a Tecnologia under grants PTDC/PSI-PCO/110734/2009, SFRH/BPD/72974/2010, EXPL/MHC-PCN/0299/2013, PEst-OE/EQB/ LA0023/2014, UID/BIM/04773/2013 CBMR 1334, and UID/PSI/00050/2013).

\section{References}

Barreto, H., Moreira, A., \& Ferreira, C. (2008). Wechsler Adult Intelligence Scale (WAIS-III): Versão Portuguesa [WAIS-III: Portuguese version]. Lisboa: Cegoc.

Bellocchi, S., Muneaux, M., Bastien-Toniazzo, M., \& Ducrot, S. (2013). I can read it in your eyes: what eye movements tell us about visuo-attentional processes in developmental dyslexia. Research in Developmental Disabilities, 34(1), 452-460. doi:10.1016/j.ridd.2012.09.002.

Blomert, L., \& Vaessen, A. (2009). 3DM differential diagnostics for dyslexia: cognitive analysis of reading and spelling. Amsterdam: Boom Test.

Bosse, M.-L., Tainturier, M. J., \& Valdois, S. (2007). Developmental dyslexia: the visual attention span deficit hypothesis. Cognition, 104(2), 198-230. doi:10.1016/j.cognition.2006.05.009.

Bouma, H. (1973). Visual interference in the parafoveal recognition of initial and final letters of words. Vision Research, 13(4), 767-782. doi:10.1016/0042-6989(73)90041-2.

Bouma, H., \& Legein, C. P. (1977). Foveal and parafoveal recognition of letters and words by dyslexics and by average readers. Neuropsychologia, 15(1), 69-80. doi:10.1016/0028-3932(77)90116-6.

Callens, M., Whitney, C., Tops, W., \& Brysbaert, M. (2013). No deficiency in left-to-right processing of words in dyslexia but evidence for enhanced visual crowding. The Quarterly Journal of Experimental Psychology, 66(9), 1803-1817. doi:10.1080/17470218.2013.766898.

Castro, S. L., \& Alves, R. A. (2005). Despistagem da dislexia em adultos através do Questionário de História de Leitura. Iberpsicología: Revista Electrónica de la Federación española de Asociaciones de Psicología, 10(8), 9-. Accessed 11 Mar 2015.

Chace, K. H., Rayner, K., \& Well, A. D. (2005). Eye movements and phonological parafoveal preview: effects of reading skill. Canadian Journal of Experimental Psychology/Revue Canadienne de Psychologie Expérimentale, 59(3), 209-217. doi:10.1037/h0087476.

De Luca, M., Di Pace, E., Judica, A., Spinelli, D., \& Zoccolotti, P. (1999). Eye movement patterns in linguistic and non-linguistic tasks in developmental surface dyslexia. Neuropsychologia, 37(12), 1407-1420. doi:10. 1016/S0028-3932(99)00038-X.

Denckla, M. B., \& Rudel, R. G. (1976). Rapid "automatized" naming (R.A.N.): dyslexia differentiated from other learning disabilities. Neuropsychologia, 14(4), 471-479. doi:10.1016/0028-3932(76)90075-0.

Di Filippo, G., Brizzolara, D., Chilosi, A., De Luca, M., Judica, A., Pecini, C., et al. (2005). Rapid naming, not cancellation speed or articulation rate, predicts reading in an orthographically regular language (Italian). Child Neuropsychology, 11(4), 349-361. doi:10.1080/09297040490916947.

Di Filippo, G., Brizzolara, D., Chilosi, A., De Luca, M., Judica, A., Pecini, C., et al. (2006). Naming speed and visual search deficits in readers with disabilities: evidence from an orthographically regular language (Italian). Developmental Neuropsychology, 30(3), 885-904. doi:10.1207/s15326942dn3003_7. 
Fernandes, T., Araújo, S., Sucena, A., Reis, A., \& Castro, S. L. (2014). The 1-min screening test for reading problems in college students: psychometric properties and dyslexic readers profile. Submitted.

Georgiou, G. K., Parrila, R., Cui, Y., \& Papadopoulos, T. C. (2013). Why is rapid automatized naming related to reading? Journal of Experimental Child Psychology, 115(1), 218-225. doi:10.1016/j.jecp.2012.10.015.

Germano, G. D., Reilhac, C., Capellini, S. A., \& Valdois, S. (2014). The phonological and visual basis of developmental dyslexia in Brazilian Portuguese reading children. Frontiers in Psychology, 5. doi:10.3389/fpsyg.2014.01169.

Hawelka, S., \& Wimmer, H. (2005). Impaired visual processing of multi-element arrays is associated with increased number of eye movements in dyslexic reading. Vision Research, 45(7), 855-863. doi:10.1016/j.visres.2004.10.007.

Hawelka, S., \& Wimmer, H. (2008). Visual target detection is not impaired in dyslexic readers. Vision Research, 48(6), 850-852. doi:10.1016/j.visres.2007.11.003.

Henderson, J. M., \& Ferreira, F. (1990). Effects of foveal processing difficulty on the perceptual span in reading: implications for attention and eye movement control. Journal of Experimental Psychology: Learning, Memory, and Cognition, 16(3), 417-429. doi:10.1037/0278-7393.16.3.417.

Jones, M. W., Kelly, M. L., \& Corley, M. (2007). Adult dyslexic readers do not demonstrate regularity effects in sentence processing: evidence from eye-movements. Reading and Writing, 20(9), 933-943. doi:10.1007/ s11145-007-9060-3.

Jones, M. W., Obregón, M., Louise Kelly, M., \& Branigan, H. P. (2008). Elucidating the component processes involved in dyslexic and non-dyslexic reading fluency: an eye-tracking study. Cognition, 109(3), 389-407. doi:10.1016/j.cognition.2008.10.005.

Jones, M. W., Branigan, H. P., \& Kelly, M. L. (2009). Dyslexic and nondyslexic reading fluency: rapid automatized naming and the importance of continuous lists. Psychonomic Bulletin \& Review, 16(3), 567572. doi:10.3758/PBR.16.3.567.

Lassus-Sangosse, D., N'guyen-Morel, M.-A., \& Valdois, S. (2008). Sequential or simultaneous visual processing deficit in developmental dyslexia? Vision Research, 48(8), 979-988. doi:10.1016/j.visres.2008.01.025.

Lewandowska, M., Milner, R., Ganc, M., Włodarczyk, E., \& Skarżyński, H. (2014). Attention dysfunction subtypes of developmental dyslexia. Medical Science Monitor: International Medical Journal of Experimental and Clinical Research, 20, 2256-2268. doi:10.12659/MSM.890969.

Martelli, M., Di Filippo, G., Spinelli, D., \& Zoccolotti, P. (2009). Crowding, reading, and developmental dyslexia. Journal of Vision, 9(4), 14. doi:10.1167/9.4.14.

McConkie, G. W., \& Rayner, K. (1975). The span of the effective stimulus during a fixation in reading. Perception \& Psychophysics, 17(6), 578-586. doi:10.3758/BF03203972.

Moll, K., \& Jones, M. (2013). Naming fluency in dyslexic and nondyslexic readers: differential effects of visual crowding in foveal, parafoveal, and peripheral vision. The Quarterly Journal of Experimental Psychology, 66(11), 2085-2091. doi:10.1080/17470218.2013.840852.

Moores, E., Cassim, R., \& Talcott, J. B. (2011). Adults with dyslexia exhibit large effects of crowding, increased dependence on cues, and detrimental effects of distractors in visual search tasks. Neuropsychologia, 49(14), 3881-3890. doi:10.1016/j.neuropsychologia.2011.10.005.

Nergård-Nilssen, T., \& Hulme, C. (2014). Developmental dyslexia in adults: behavioural manifestations and cognitive correlates. Dyslexia, 20(3), 191-207. doi:10.1002/dys.1477.

Norton, E. S., \& Wolf, M. (2012). Rapid automatized naming (RAN) and reading fluency: implications for understanding and treatment of reading disabilities. Annual Review of Psychology, 63, 427-452. doi:10. 1146/annurev-psych-120710-100431.

Pavlidis, G. T. (1981). Do eye movements hold the key to dyslexia? Neuropsychologia, 19(1), 57-64.

Perea, M., Panadero, V., Moret-Tatay, C., \& Gómez, P. (2012). The effects of inter-letter spacing in visual-word recognition: evidence with young normal readers and developmental dyslexics. Learning and Instruction, 22(6), 420-430. doi:10.1016/j.learninstruc.2012.04.001.

Pernet, C., Valdois, S., Celsis, P., \& Démonet, J.-F. (2006). Lateral masking, levels of processing and stimulus category: a comparative study between normal and dyslexic readers. Neuropsychologia, 44(12), 2374-2385. doi:10.1016/j.neuropsychologia.2006.05.003.

Pollatsek, A., Reichle, E. D., \& Rayner, K. (2006). Tests of the E-Z Reader model: exploring the interface between cognition and eye-movement control. Cognitive Psychology, 52(1), 1-56. doi:10.1016/j.cogpsych.2005.06.001.

Rayner, K., Murphy, L. A., Henderson, J. M., \& Pollatsek, A. (1989). Selective attentional dyslexia. Cognitive Neuropsychology, 6(4), 357-378. doi:10.1080/02643298908253288.

Reichle, E. D., Rayner, K., \& Pollatsek, A. (2003). The E-Z reader model of eye-movement control in reading: comparisons to other models. The Behavioral and Brain Sciences, 26(4), 445-476. discussion 477-526.

Reis, A., Faísca, L., Castro, S., Inácio, F., Pacheco, A., Araújo, S., \& Santos, M. (2014). Versão Portuguesa da Bateria 3DM para avaliação da leitura e da escrita [3DM Portuguese version to assess reading and spelling skills]. Manuscript in preparation.

Schotter, E. R., Angele, B., \& Rayner, K. (2012). Parafoveal processing in reading. Attention, Perception, \& Psychophysics, 74(1), 5-35. doi:10.3758/s13414-011-0219-2. 
Sperlich, A., Schad, D. J., \& Laubrock, J. (2015). When preview information starts to matter: development of the perceptual span in German beginning readers. Journal of Cognitive Psychology, 5, 1-20. doi:10.1080/ 20445911.2014.993990.

Spinelli, D., De Luca, M., Judica, A., \& Zoccolotti, P. (2002). Crowding effects on word identification in developmental dyslexia. Cortex, 38(2), 179-200. doi:10.1016/S0010-9452(08)70649-X.

Sucena, A., \& Castro, S. L. (2010). Aprender a ler e avaliar a leitura. O TIL: Teste de Idade de Leitura. Coimbra: Almedina.

Valdois, S., Bosse, M.-L., \& Tainturier, M.-J. (2004). The cognitive deficits responsible for developmental dyslexia: review of evidence for a selective visual attentional disorder. Dyslexia (Chichester, England), 10(4), 339-363. doi:10.1002/dys.284.

Wechsler, D. (1997). WAIS-III, wechsler adult intelligence scale: administration and scoring manual. Psychological Corporation.

Wimmer, H., Mayringer, H., \& Landerl, K. (1998). Poor reading: a deficit in skill-automatization or a phonological deficit? Scientific Studies of Reading, 2(4), 321-340. doi:10.1207/s1532799xssr0204 2.

Wolf, M., \& Bowers, P. G. (1999). The double-deficit hypothesis for the developmental dyslexias. Journal of Educational Psychology, 91(3), 415-438. doi:10.1037/0022-0663.91.3.415.

Yan, M., Pan, J., Laubrock, J., Kliegl, R., \& Shu, H. (2013). Parafoveal processing efficiency in rapid automatized naming: a comparison between Chinese normal and dyslexic children. Journal of Experimental Child Psychology, 115(3), 579-589. doi:10.1016/j.jecp.2013.01.007.

Ziegler, J. C., Pech-Georgel, C., Dufau, S., \& Grainger, J. (2010). Rapid processing of letters, digits and symbols: what purely visual-attentional deficit in developmental dyslexia? Developmental Science, 13(4), F8-F14. doi:10.1111/j.1467-7687.2010.00983.x.

Zoccolotti, P., De Luca, M., Lami, L., Pizzoli, C., Pontillo, M., \& Spinelli, D. (2013). Multiple stimulus presentation yields larger deficits in children with developmental dyslexia: a study with reading and RAN-type tasks. Child Neuropsychology, 19(6), 639-647. doi:10.1080/09297049.2012.718325.

Zorzi, M., Barbiero, C., Facoetti, A., Lonciari, I., Carrozzi, M., Montico, M., et al. (2012). Extra-large letter spacing improves reading in dyslexia. Proceedings of the National Academy of Sciences, 109(28), 1145511459. doi:10.1073/pnas.1205566109. 